Pacific Journal of Mathematic 


\title{
CANONICAL MODELS FOR INVARIANT SUBSPACES
}

\author{
Michael McAsey
}

\begin{abstract}
The invariant subspace structure of an operator algebra $\mathfrak{Z}_{+}$is completely determined. The non-selfadjoint algebra is constructed from a cyclic transformation acting on a finite set. There is a distinguished (finite) set of invariant subspaces of $\mathfrak{Z}_{+}$which has been identified elsewhere. These subspaces are used as canonical models; all other invariant subspaces for $\mathfrak{L}_{+}$ are described in terms of these subspaces. Uniqueness of this representation is also discussed.
\end{abstract}

1. Introduction. This paper is a continuation of [5]. While some acquaintance with [5] would be helpful in reading the present paper, it is not absolutely necessary. Here we determine completely the invariant subspace structure of an operator algebra $\mathfrak{L}_{+}$constructed from a cyclic transformation on a finite set. (The definitions of $\mathbb{S}_{+}$and an allied algebra $\Re_{+}$are given below.) As shown in [5] there is a distinguished class of subspaces invariant for both $\mathfrak{L}_{+}$and $\mathfrak{R}_{+}$. We shall show that the invariant subspaces for $\mathfrak{L}_{+}$ can be completely described in terms of these distinguished subspaces in much the same spirit as Buerling's theorem describes the invariant subspaces of the shift.

To define the algebras $\mathfrak{L}_{+}$and $\Re_{+}$, let $X$ be a finite set with elements $x_{0}, x_{1}, \cdots, x_{n-1}$ and let $\tau$ be the permutation of $X$ defined by $\tau\left(x_{i}\right)=x_{i+1}(i \neq n-1)$ and $\tau\left(x_{n-1}\right)=x_{0}$. Let $Z$ denote the set of integers and let $l^{2}(\boldsymbol{Z} \times X)$ be the Hilbert space of all (complex-valued) functions $f$ on $\boldsymbol{Z} \times X$ such that $\sum_{n} \sum_{x}|f(n, x)|^{2}<\infty$. Let $f$ be an element of $l^{2}(\boldsymbol{Z} \times X)$ and define operators $L_{\hat{o}}$ and $R_{\hat{o}}$ on $l^{2}(\boldsymbol{Z} \times X)$ as follows:

$$
\left(L_{\hat{\sigma}} f\right)(n, x)=f\left(n-1, \tau^{-1} x\right) ;\left(R_{i} f\right)(n, x)=f(n-1, x) .
$$

For a complex-valued function $\varphi$ defined on $X$ (i.e., $\varphi \in l^{\infty}(X)$ ), we define operators $L_{\varphi}$ and $R_{\varphi}$ on $l^{2}(\boldsymbol{Z} \times X)$ :

$$
\left(L_{\varphi} f\right)(n, x)=\varphi(x) f(n, x) ;\left(R_{\varphi} f\right)(n, x)=\varphi\left(\tau^{-n} x\right) f(n, x) .
$$

Let $\mathfrak{M}_{L}=\left\{L_{\varphi} \mid \varphi \in l^{\infty}(X)\right\}$ and $\mathfrak{M}_{R}=\left\{R_{\varphi} \mid \varphi \in l^{\infty}(X)\right\}$. The algebra \& (resp. $\Re$ ) is defined to be the von Neumann algebra generated by $L_{\hat{o}}$ and $\mathfrak{M}_{L}$ (resp. $R_{\hat{o}}$ and $\mathfrak{M}_{R}$ ). Finally we define the non-selfadjoint algebra $\mathfrak{L}_{+}$(resp. $\mathfrak{R}_{+}$) to be the weakly closed algebra generated by $L_{\mathfrak{s}}$ and $\mathfrak{M}_{L}$ (resp. $R_{\hat{o}}$ and $\mathfrak{M}_{R}$ ).

The algebras $\mathbb{R}$ and $\mathfrak{R}$ are crossed products and $\mathfrak{L}_{+}$and $\mathfrak{R}_{+}$are called non-selfadjoint crossed products. We refer the reader to 
$[5,6]$ for discussions of these algebras including some of their elementary properties. It should be noted that one of the principal results of [5] identifies the equivalence classes of $\mathbb{L}_{+}$-invariant subspaces that are unitarily equivalent by a unitary operator in $\Re$. Each such class can also be described in terms of a multiplicity function. These functions play an important role in this paper (cf. $\S 3)$ for, as we shall see, the multiplicity function will allow us to identify a canonical subspace in each equivalence class of $\mathfrak{L}_{+}$-invariant subspaces.

It is shown in [5] that for certain subsets $B$ of $Z \times X$, subspaces of the form $l^{2}(B)=\left\{f \in l^{2}(Z \times X) \mid f(n, x)=0\right.$ for $\left.(n, x) \notin B\right\}$ are invariant under both the algebras $\mathfrak{L}_{+}$and $\Re_{+}$. Such a set $B$ must be invariant under the two maps $\lambda$ and $\rho$ defined on $Z \times X$ as follows:

$$
\begin{aligned}
& \lambda(n, x)=(n+1, \tau x) \\
& \rho(n, x)=(n+1, x) .
\end{aligned}
$$

In case $\tau$ is a nonperiodic transformation on a measure space $X$ as discussed in [5], all $\mathfrak{R}_{+} \vee \Re_{+}$-invariant subspaces have the form $l^{2}(B)$. As shown in $\S 5$ of [5], this is not the case when $\tau$ is a periodic transformation acting on a finite discrete set, as considered here.

In this paper we shall show that the $\mathfrak{L}_{+} \vee \Re_{+}$-invariant subspaces of the form $l^{2}(B)$ are sufficiently plentiful to allow us to describe all the pure $\mathbb{S}_{+}$-invariant subspaces in terms of these more elementary ones. (A pure subspace is one that contains no nonzero subspace reducing $\mathfrak{Q}_{+}$) The idea is to use subspaces of the form $l^{2}(B)$ as canonical models for the $\mathbb{L}_{+}$-invariant subspaces. This concept is made precise in the following definition. (For a subspace $\mathscr{M}, P_{\mathscr{M}}$ denotes the orthogonal projection onto $\mathscr{C}$.)

DEFINITION. A family of full, pure invariant subspaces $\left\{\mathscr{A}_{i}\right\}_{i \in I}$ constitutes a complete set of canonical models for the pure $\mathfrak{L}_{+}$ invariant subspaces in case (a) for no two distinct indices $i$ and $j_{i}^{\text {' }}$ is $P_{\mathscr{N}_{i}}$ unitarily equivalent to $P_{\mathscr{N}_{j}}$ by a unitary operator in $\Re$; and (b) for every pure $\mathscr{L}_{+}$-invariant subspace $\mathscr{C}$ there is an $i$ in $I$ and a partial isometry $R_{\theta}$ in $\Re$ such that $R_{\theta} P_{\| i} R_{\|}^{*}=P_{\mathscr{M}}$.

In particular the last equation implies that $\mathscr{C}=R_{\theta} \mathscr{M}_{i}$. The motivation for this concept stems from Beurling's theorem. Recall that this theorem states that if $\mathscr{M}$ is a (nonzero) nonreducing subspace for the bilateral shift on $L^{2}$ (of the unit circle), then $\mathscr{M}=$ $\theta H^{2}$ where $\theta$ is a unimodular function on the circle. In this case 
the singleton $\left\{H^{2}\right\}$ is a complete set of canonical models for the pure invariant subspaces of the shift. A similar result is valid if we consider the non-selfadjoint crossed product determined by a factor (of a special kind) and an automorphism implemented by a unitary operator acting on the factor. In this case also a single subspace, $l^{2}\left(\boldsymbol{Z}_{+}, \mathscr{H}\right)$, forms a complete set of canonical models for the invariant subspaces of the algebra $\mathbb{S}_{+}$as shown in [6]. It is shown in [5], with the particular algebra $\mathfrak{L}_{+}$as we have defined it here, that there are pure subspaces $\mathscr{C}$ and $\mathscr{N}$ invariant for $\mathfrak{L}_{+}$for which there is no partial isometry $R_{\theta}$ in $\Re$ satisfying $R_{\theta} P_{\mathscr{M}} R_{\theta}^{*}=P_{\mathscr{S}}$; thus a set of canonical models will necessarily consist of more than one subspace in the situation considered here. In this paper it will be shown that a finite collection of subspaces of the form $l^{2}(B)$ provides a complete set of canonical models for the (pure) $\mathbb{Z}_{+}$-invariant subspaces. Moreover, it will be seen that in this case the multiplicity function provides the necessary information for explicitly constructing the subspace from the canonical model.

Taking into account the results of [5], this is what we will have accomplished: we will have classified the invariant subspaces of $\mathfrak{S}_{+}$up to a specific kind of equivalence and we will have identified a canonical member of each equivalence class. Moreover we will have done this in a fashion which is identical in spirit with that exhibited in two other contexts in which reasonably successful generalizations of the theory of shifts have been found, namely, the theory of invariant subspaces on multiply connected domains [1] and the theory of compact groups with ordered duals [4].

2. Alternative representations of $\mathfrak{L}$ and $\Re$. The algebras $\mathbb{Z}$ and $\Re$ have been discussed in [5] under different hypotheses on $X$ and $\tau$. One major difference between the algebras $\mathcal{L}$ and $\Re$ considered in $\S 4$ of [5] and those in this paper is that in [5] \& and $\Re$ are factors while here they are not factors. Indeed a computation using the periodicity of $\tau$ shows that $L_{\hat{o}}^{n}=R_{\tilde{o}}^{n}$ where, recall, $n$ is the cardinality of the set $X$. Since $\&$ and $\Re$ are commutants of one another, it follows that $\&$ and $\Re$ have nontrivial centers and so are not factors. As a consequence of the representation developed in this section, we show that the center of $\&$ (and of $\Re$ ) is the von Neumann algebra generated by $L_{\delta}^{n}$.

In this section we shall show how the algebras $\mathbb{L}$ and $\Re$ may be viewed as the left and right regular representations of the algebra of all $n \times n$-matrices with entries from $L^{\infty}(\boldsymbol{T})$, the (essentially) bounded functions on the circle. In order to do this, an isomorphism will be constructed between the spaces $l^{2}(\boldsymbol{Z} \times X)$ and $L^{2}(\boldsymbol{T}) \otimes M_{n}$. This second space will be viewed as the set of $n \times n$ - 
matrices with entries from $L^{2}(\boldsymbol{T})$. It is a Hilbert space with inner product

$$
\left(\left[f_{i j}\right],\left[g_{i j}\right]\right)=\sum_{i, j} \int_{T} f_{i j}(x) g_{i j}(x) d x
$$

for elements $\left[f_{i j}\right]$ and $\left[g_{i j}\right]$ in $L^{2}(\boldsymbol{T}) \otimes \boldsymbol{M}_{n}$. (The norm on $\boldsymbol{M}_{n}$ here is the Hilbert-Schmidt norm.) The algebra $\mathbb{L}$ (resp. $\Re$ ) will be realized as the algebra $L^{\infty}(\boldsymbol{T}) \otimes M_{n}$ acting by left (resp. right) multiplication on the space $L^{2}(\boldsymbol{T}) \otimes \boldsymbol{M}_{n}$.

The isomorphism mentioned above will be constructed by mapping one basis to another. The set $\mathscr{B}_{1}$ of indicator (or characteristic) functions of singletons $\left\{1_{\{(n, x)\}} \mid n \in Z, x \in X\right\}$ is an orthonormal basis for the space $l^{2}(\boldsymbol{Z} \times X)$. For the other basis, let $E_{i j}$ be the $n \times n$ matrix whose only nonzero entry is a 1 in the $(i, j)$ th position. Let $\chi_{n}$ be the function on the circle defined by $\chi_{n}(z)=z^{n}$. The set $\mathscr{B}_{2}=$ $\left\{\chi_{k} E_{i j} \mid i, j=1,2, \cdots, n ; k \in Z\right\}$ is an orthonormal basis for the Hilbert space $L^{2}(\boldsymbol{T}) \otimes \boldsymbol{M}_{n}$. (We assume that the measure on the circle is normalized Lebesgue measure.)

There are several ways to define the map $W$ from $\mathscr{B}_{1}$ to $\mathscr{B}_{2}$. For an easy way, first let $\delta$ be the function defined on $Z \times X$ by $\delta(n, x)=\left\{\begin{array}{ll}1 & n=1 \\ 0 & n \neq 1\end{array}\right.$ and define

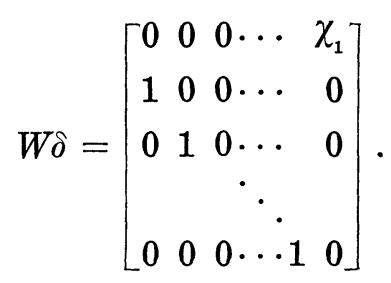

Definition. The map $W: \mathscr{B}_{1} \rightarrow \mathscr{B}_{2}$ is defined first on basis elements of the form $1_{\left\{\left(0, x_{i}\right)\right\}}: W 1_{\left\{\left(0, x_{0}\right)\right\}}=E_{n n}, W 1_{\left\{\left(0, x_{i}\right)\right\}}=E_{i i}(i \neq 0)$. For other elements of $\mathscr{B}_{1}$ let

$$
W 1_{\left\{\left(k, x_{i}\right)\right\}}=W 1_{\left\{\left(0, x_{i}\right)\right\}}(W \delta)^{k} .
$$

Observe that the operator $L_{\delta}$ (resp. $R_{\delta}$ ) acting on the basis $\mathscr{B}_{1}$ of $l^{2}(\boldsymbol{Z} \times X)$ is transformed into left (resp. right) multiplication by $W \delta$ on $L^{2}(\boldsymbol{T}) \otimes \boldsymbol{M}_{n}$.

The action of $W$ on the bases can be extended to a Hilbert space isomorphism from $l^{2}(\boldsymbol{Z} \times X)$ to $L^{2}(\boldsymbol{T}) \otimes \boldsymbol{M}_{n}$. The map $W$ has one other important feature: it is multiplicative on the bases on which it is defined. Let $L_{0}^{2}=\left\{f \in l^{2}(Z \times X) \mid f(n, x)=0\right.$ for all but finitely many $n$ \}. For elements $f$ and $g$ in $L_{0}^{2}$, we define a multiplication as follows: 


$$
(f * g)(n, x)=\sum_{k=-\infty}^{\infty} f(k, x) g\left(n-k, \tau^{-k} x\right) .
$$

The bounded elements of $l^{2}(\boldsymbol{Z} \times X)$ are defined to be those functions $f$ such that $g \mapsto f * g$ is a bounded operation on $L_{0}^{2}$. The set of bounded elements is denoted by $L^{\infty}$. Note that by definition $L^{\infty} \cong$ $l^{2}(\boldsymbol{Z} \times X)$. (Our notation coincides with that in [6].) An example of a bounded element is the function $\delta$ defined above. A computation shows that $g \mapsto \delta * g$ (resp. $g \mapsto g * \delta$ ) is simply the operator $L_{\hat{o}}$ (resp. $R_{\delta}$ ) acting on $g$. It is shown in [5] that the algebra of left (resp. right) multiplications by elements of $L^{\infty}$ is precisely the algebra $\&$ (resp. $\Re$ ). Accordingly we shall use the notation $L_{f}$ for left multiplication by an element $f$ in $L^{\infty}$. Although slightly tedious, the proof of the following theorem is not difficult and is left to the reader.

THEOREM 2.1. The map $W$ is a Hilbert space isomorphism from $l^{2}(\boldsymbol{Z} \times X)$ onto $L^{2}(\boldsymbol{T}) \otimes M_{n}$ such that $W L_{f} W^{-1}=L_{W f}$ and $W R_{f} W^{-1}=R_{W f}$ for $f$ in $L^{\infty}$.

Since $W$ is an isomorphism, it follows immediately that $W \& W^{-1}$ is a von Neumann subalgebra of the bounded operators on $L^{2}(\boldsymbol{T}) \otimes$ $\boldsymbol{M}_{n}$. This subalgebra is identified in the following corollary. (From now on, most results will be stated for the left algebra \&; corresponding statements for the algebra $\Re$ will be left to the reader.)

CoROLLARY 2.2. The algebra $W \Omega W^{-1}$ is isomorphic to the algebra $L^{\infty}(\boldsymbol{T}) \otimes \boldsymbol{M}_{n}$ acting by left multiplication on $L^{2}(\boldsymbol{T}) \otimes \boldsymbol{M}_{n}$.

Proof. Recall that we treat $L^{p}(\boldsymbol{T}) \otimes M_{n}$ as the set of $n \times n$ matrices with entries from $L^{p}(\boldsymbol{T})$. Consider the subalgebra $\Omega_{0}$ of $\&$ defined by $\Omega_{0}=\left\{L_{f} \mid f \in L_{0}^{2}\right\}$. Then $W \Omega_{0} W^{-1}$ is the algebra of $n \times n$-matrices with "polynomial" entries acting by left multiplication on the space $L^{2}(\boldsymbol{T}) \otimes \boldsymbol{M}_{n}$. So for $L_{f}$ in $\Omega_{0}, W L_{f} W^{-1}$ is left multiplication by a matrix $\left[p_{i j}\right]$ where for each $i$ and $j, p_{i j}$ is a polynomial of the form $\sum_{l=-K}^{K} a_{l} \chi_{l}\left(a_{l} \in C\right)$. The weak closure of $W \Omega_{0} W^{-1}$ is $W \& W^{-1}$ and thus $W \& W^{-1}$ is simply the algebra $L^{\infty}(\boldsymbol{T}) \otimes M_{n}$ thought of as acting by left multiplication on $L^{2}(\boldsymbol{T}) \otimes$ $\boldsymbol{M}_{n}$.

Since the algebra $\&$ is a generalization of the algebra $L^{\infty}(\boldsymbol{T})$ and since $\mathscr{R}_{+}$is designed to generalize $H^{\infty}(\boldsymbol{T})$, the subspace of $L^{\infty}(\boldsymbol{T})$ consisting of functions whose Fourier coefficients of negative index vanish, it is tempting to say that $W \Omega_{+} W^{-1}$ is the algebra $H^{\infty}(\boldsymbol{T}) \otimes$ $\boldsymbol{M}_{n}$. However, as the next two results show, this is not quite the case. 
COROLLARY 2.3. The algebra $W \Omega_{+} W^{-1}$ consists of the matrices $\left[a_{i j}\right]$ in $H^{\infty}(\boldsymbol{T}) \otimes \boldsymbol{M}_{n}$ having the property that $\hat{a}_{i j}(0)=0$ if $a_{i j}$ lies above the main diagonal. That is,

$$
W \Omega_{+} W^{-1}=\left[\begin{array}{cccc}
H^{\infty} & H_{0}^{\infty} & \cdots & H_{0}^{\infty} \\
H^{\infty} & H^{\infty} & \cdots & H_{0}^{\infty} \\
\vdots & \ddots & \vdots \\
H^{\infty} & H^{\infty} & \cdots & H^{\infty}
\end{array}\right]
$$

where $H_{0}^{\infty}=\left\{f \in H^{\infty} \mid \hat{f}(0)=0\right\}$.

Proof. Since $\mathbb{L}_{+}=\left\{L_{f} \mid f \in L^{\infty}, f(n, \cdot)=0, n<0\right\}$ and $L^{\infty} \cong l^{2}(Z \times$ $X)$, any function $f$ such that $L_{f}$ is in $\mathscr{Q}_{+}$is also in $l^{2}\left(\boldsymbol{Z}_{+} \times X\right)$. $\left(\boldsymbol{Z}_{+}=\{0,1,2, \cdots\}\right.$.) Using the definition of $W \delta$ and the form of $(W \delta)^{k}$ for $k \geqq 0$, it follows that $W \Omega_{+} W^{-1} \cong H^{\infty}(\boldsymbol{T}) \otimes \boldsymbol{M}_{n}$.

To see that $W \Omega_{+} W^{-1}$ has the stated matricial form, it suffices to show that if $j \geqq 0$ and $W 1_{\left\{\left(j, x_{l}\right)\right\}}=\chi_{k} E_{\alpha \beta}$ with $\alpha<\beta$ then $k$ is strictly greater than zero. But $W 1_{\left\{\left(j, x_{l}\right)\right\}}=E_{p p}(W \delta)^{j}$ where $p=l$ if $l \neq 0$ and $p=n$ if $l=0$. For $j \geqq 0$, the nonzero elements on or above the main diagonal of $(W \delta)^{j}$ are positive powers of $\chi_{1}$. Since $\chi_{1}^{k}=\chi_{k}$, the result follows.

REMARK 2.4. (1) In view of 2.3, it is clear that the algebras $W \Omega_{+} W^{-1}$ and $H^{\infty}(\boldsymbol{T}) \otimes \boldsymbol{M}_{n}$ should not be isomorphic. The following proof that they, in fact, are not isomorphic is due to K. R. Fuller. In this remark, $H^{\infty}$ will always be used to denote $H^{\infty}(\boldsymbol{T})$. Note first that $H_{0}^{\infty}$ is just the principal ideal $z H^{\infty}$. Let

$$
J=\left[\begin{array}{cccc}
H_{0}^{\infty} & H_{0}^{\infty} & \cdots & H_{0}^{\infty} \\
H^{\infty} & H_{0}^{\infty} & \cdots & H_{0}^{\infty} \\
\vdots & \ddots & \vdots \\
H^{\infty} & H^{\infty} & \cdots & H_{0}^{\infty}
\end{array}\right]
$$

Then $J$ is a (two-sided) ideal in $W \&_{+} W^{-1}$ and $W \&_{+} W^{-1} / J$ is the ring

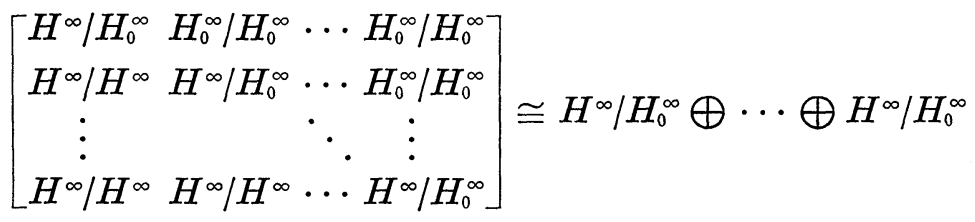

( $n$ summands). Thus $W \mathbb{R}_{+} W^{-1}$ has a commutative factor ring. Recall that any (two-sided) ideal in $H^{\infty} \otimes \boldsymbol{M}_{n}$ has the form $I \otimes \boldsymbol{M}_{n}$ where $I$ is an ideal in $H^{\infty}$. It follows that $H^{\infty} \otimes M_{n}$ has no com- 
mutative factor rings. Hence $H^{\infty} \otimes \boldsymbol{M}_{n}$ is not isomorphic to $W \Re_{+} W^{-1}$.

(2) It was noted at the beginning of this section that the algebras $\mathbb{Z}$ and $\mathfrak{R}$ are not factors. We now show how to identify $\mathscr{Z}(\mathbb{R})$, the center of $\mathbb{R}$, as the von Neumann algebra generated by $L_{\delta}^{n}$. To do this we first write $L_{M_{n}}$ for the von Neumann algebra of left multiplications by $n \times n$-matrices acting on $\boldsymbol{M}_{n}$, and similarly write $R_{M_{n}}$ for the right multiplications. Let $C_{*}$ denote the algebra of scalar multiples of the identity acting on the space $\mathscr{H}$. Then Corollary 2.3 shows that $\mathfrak{Q} \cong L^{\infty}(\boldsymbol{T}) \otimes L_{M_{n}}$ and $\mathfrak{Q}^{\prime}=\Re \cong L^{\infty}(\boldsymbol{T}) \otimes R_{M_{n}}$. Hence $\mathscr{L}(\mathbb{L})=\mathbb{R} \cap \mathbb{L}^{\prime} \cong\left(L^{\infty}(\boldsymbol{T}) \otimes L_{M_{n}}\right) \cap\left(L^{\infty}(\boldsymbol{T}) \otimes R_{M_{n}}\right)=L^{\infty}(\boldsymbol{T}) \otimes\left(L_{M_{n}} \cap\right.$ $\left.R_{M_{n}}\right)=L^{\infty}(\boldsymbol{T}) \otimes C_{M_{n}} \cong\left\{L_{i}^{n}\right\}^{\prime \prime}$.

3. Two-sided invariant subspaces and multiplicity functions. Recall that a subspace that is invariant under both the algebras $\mathfrak{L}_{+}$and $\Re_{+}$is called a two-sided invariant or $\mathfrak{L}_{+} \vee \Re_{+}$invariant subspace. Under appropriate assumptions on $X$ and $\tau$, it can be shown that for each two-sided invariant subspace $\mathscr{C}$ there is a subset $B$ of $Z \times X$ invariant for the maps $\lambda$ and $\rho$ defined in $\S 1$; the subspace $\mathscr{C}$ consists precisely of those functions in the Hilbert space with support contained in $B$. As noted previously, this result is not valid in case $X$ is a finite discrete set and $\tau$ is a permutation. However, it is an easy computation to show that for any set $B$ in $Z \times X$ invariant for $\lambda$ and $\rho$, the subspace $l^{2}(B)$ is a two-sided invariant subspace. The following remark collects several pertinent observations concerning two-sided invariant subspaces of the form $l^{2}(B)$.

REMARK 3.1. Let $l^{2}(B)$ be a (nontrivial) two-sided invariant subspace.

(1) If the point $(k, x)$ is in $B$ then, by the $\rho$-invariance of $B,(k+l, x)$ is in $B$ for any positive integer $l$.

(2) Let $\boldsymbol{Z}_{(k)}=\{k, k+1, k+2, \cdots\}$. There exists an integer $N$ with the property that the set $Z_{(N)} \times X$ contains $B$. This follows from the fact that $l^{2}(B)$ is not the entire space $l^{2}(Z \times X)$ and that $B$ is invariant for both maps $\lambda$ and $\rho$. If $N$ is also chosen to be the largest such integer, then $B$ contains the set $\boldsymbol{Z}_{(N+n)} \times X$. In particular $l^{2}(B)$ is a full, pure subspace for both the algebras $\mathfrak{L}_{+}$ and $\Re_{+}$. (Recall, an $\mathfrak{L}_{+}$-invariant subspace $\mathscr{C}$ is $\mathbb{L}_{+}$-full in case the smallest $\mathbb{Q}_{+}$-reducing subspace containing $\mathscr{C}$ is $l^{2}(\boldsymbol{Z} \times X)$.)

(3) There exists a partition $\left\{E_{k}\right\}_{k=-\infty}^{\infty}$ of $X$ such that $B=$ $\bigcup_{k \in \boldsymbol{Z}} \boldsymbol{Z}_{(k)} \times E_{k}$. To construct this partition, let $C=B \backslash \rho(B)$. Then $E_{k}=\{x \in X \mid(k, x) \in C \cap(\{k\} \times X)\}$. Observe that all but finitely many of the sets $E_{k}$ are empty. Since $B$ is invariant for $\lambda$, the partition 
$\left\{E_{k}\right\}_{k}$ has the following "order" property: $\tau\left(E_{k}\right) \subseteq \bigcup_{j \leqq k+1} E_{j}$. This property will be important in our discussion of multiplicity functions in Theorem 3.4.

(4) Recall that if $\mathscr{C}$ is a left-invariant subspace, then $\mathscr{F}=$ $\mathscr{C} \ominus L_{\delta} \mathscr{C}$ is a wandering subspace for $L_{\delta}$. From Lemma 3.2 of [5], we know that the projection $P_{\mathscr{F}}$ onto $\mathscr{F}$ lies in $\mathfrak{M}_{L}^{\prime}$, the commutant of $\mathfrak{M}_{L}$. Hence $P_{\mathscr{S}}$ can be written as $P_{\mathscr{F}}=\sum_{x \in X} P(x)$ where each $P(x)$ is a projection in $\mathscr{L}\left(l^{2}(Z \times\{x\})\right)$. The multiplicity function of the subspace $\mathscr{M}$ is the function $m$ on $X$ defined by

$$
m(x)=\operatorname{rank} P(x)=\text { dimension of range of } P(x) .
$$

Proposition 3.2. Let $l^{2}(B)$ be a (nontrivial) two-sided invariant subspace. Then there exists a set $A \subset B$ such that $l^{2}(A)$ is the wandering subspace for the operator $L_{\delta}$ associated with the subspace $l^{2}(B)$.

Proof. The wandering subspace is by definition $\mathscr{F}=l^{2}(B) \Theta$ $L_{\hat{\delta}} l^{2}(B)=l^{2}(B) \ominus l^{2}(\lambda(B))=l^{2}(B \backslash \lambda(B))$. Let $A=B \backslash \lambda(B)$.

In the terminology of ergodic theory, the set $A$ is a wandering subset of $Z \times X$ for the transformation $\lambda$. We have found it helpful to represent subspaces of the form $l^{2}(B)$ by means of figures. Since these subspaces consist of functions supported on the set $B$, all information concerning such a subspace is codified in the set $B$. To graph such a set, one may simply represent the set $Z \times X$ in the obvious way as the set $Z \times\{0,1,2, \cdots, n-1\}$ in the plane. The graphical representation of subsets $B$ in $Z \times X$ can be used to illustrate the items of Remark 3.1 and Proposition 3.2 as well as provide motivation for proofs of several theorems presented here.

Proposition 3.3. Assume $l^{2}(B)$ is as in 3.2 and has the multiplicity function $m$. Then $\sum_{x \in X} m(x)=n$, the cardinality of the set $X$.

Proof. Let $A$ be the wandering set produced in 3.2. For an element $x$ in $X$, define the set $A_{x}$ to be the intersection of the set $A$ and the "horizontal" slice through the point $x: A_{x}=A \cap(Z \times\{x\})$.

Let $P$ be the projection onto the wandering subspace $l^{2}(A)$. We may write $P=\sum_{x \in X} P(x)$ as in 3.1.4. Clearly $P(x)$ is the projection onto $l^{2}\left(A_{x}\right)$ for each $x$ and so $\operatorname{rank} P(x)=\operatorname{dim} l^{2}\left(A_{x}\right)=\operatorname{card} A_{x}$, the cardinality of the set $A_{x}$. Thus it suffices to show that $\sum_{x \in X}$ card $A_{x}=n$.

Using Remark 3.1.2, there exists a smallest integer $k_{0}$ such that $\left\{k_{0}\right\} \times X$ is contained in $B$. For any $x$ there exists a positive integer 
$j=j(x)$ such that $\lambda^{-j}\left(k_{0}, x\right)$ is in $B$ but $\lambda^{-(j+1)}\left(k_{0}, x\right)$ is not in $B$. By left invariance and the definition of the wandering set $A$, the point $\lambda^{-j}\left(k_{0}, x\right)=\left(k_{0}-j, \tau^{-j} x\right)$ lies in $A$. Thus associated with the $n$ distinct points $\left(k_{0}, x\right),\left(k_{0}, x_{1}\right), \cdots,\left(k_{0}, x_{n-1}\right)$, we have their pre-images under $\lambda$ in $A$ and these pre-images must be distinct. This shows that $\sum_{x \in X}$ card $A_{x} \geqq n$. But since $L_{\delta}$ is a shift of multiplicity $n$, it follows (cf. [7]) that the dimension of the wandering subspace is no larger than $n$ and hence $\sum_{x \in X}$ card $A_{x}=n$.

It is shown next that if $m$ is any nonnegative integer-valued function on $X$ whose values sum to $n$, then $m$ is a multiplicity function. Moreover we construct an explicit subspace for such a function.

TheOREM 3.4. Let $m$ be a function on $X$ having values in the nonnegative integers. If $m$ has the property that $\sum_{x \in X} m(x)=n$, then there exists a two-sided invariant subspace with multiplicity function $m$.

Proof. The proof is somewhat lengthy and is broken into three steps. The first step consists of constructing a partition $\left\{E_{k}\right\}_{k=-\infty}^{\infty}$ of $X$, in which all but finitely many of the sets $E_{k}$ will be empty. We define the set $B$ to be $\bigcup_{k \in Z} \boldsymbol{Z}_{(k)} \times E_{k}$. The second step will be to show that the set $B$ is invariant for the maps $\lambda$ and $\rho$. This invariance can be translated into a property of the partition which is then verified. The final step consists of showing that the subspace $l^{2}(B)$ has the desired multiplicity function.

Step 1. Let $\left\{x_{i_{0}}, x_{i_{1}}, \cdots, x_{i_{L}}\right\}$ be the support of $m$ so that $m(x) \neq$ 0 if and only if $x=x_{i_{k}}$ for some $k, 0 \leqq k \leqq L$. We will assume $i_{0}<i_{1}<\cdots<i_{L}$. Define the supplementary function $s(\cdot)$ on the support by

$$
s\left(x_{i_{k}}\right)=\left\{\begin{array}{cl}
0 & k=0 \\
i_{k}-i_{0}-\sum_{j=1}^{k} m\left(x_{i_{j}}\right) & k \neq 0 .
\end{array}\right.
$$

Let $s=\min \left\{s\left(x_{i_{k}}\right) \mid k=0,1, \cdots, L\right\}$. We can extend the function $s(\cdot)$ to all of $X$ as follows. For $x$ outside the support of the multiplicity function, there exists a smallest positive integer $k$ such that $x=\tau^{k} y$ with $y \in \operatorname{supp} m$. For such $x$ define $s(x)=s(y)+k$.

Define the sets $E_{k}=\{x \in X \mid s(x)=s+k\}, k=0,1, \cdots, \eta$. Clearly $\left\{E_{k}\right\}_{k=0}^{\eta}$ is a partition of $X$ where $\eta$ is the smallest positive integer such that $\bigcup_{k=0}^{n} E_{k}=X$. Let $B=\bigcup_{k=0}^{n} \boldsymbol{Z}_{(k)} \times E_{k}$. Then $l^{2}(B)=$ $\sum_{k=0}^{\eta} l^{2}\left(\boldsymbol{Z}_{(k)} \times E_{k}\right)$ is a subspace contained in $l^{2}\left(\boldsymbol{Z}_{+} \times X\right)$. 
Step 2. Clearly $l^{2}(B)$ is invariant for the right algebra $\Re_{+}$. To show the subspace is invariant for $\mathfrak{Q}_{+}$, it suffices to show that $B$ is invariant for the map $\lambda$. To accomplish this last objective, it suffices to show that the partition has the following "order" property:

$$
\tau\left(E_{k}\right) \subseteq \bigcup_{j \leqq k+1} E_{j}, \quad k=0,1,2 \cdots
$$

Finally, it follows from the definition of the partition that this property will be demonstrated once we show $s(\tau(x)) \leqq s(x)+1$. The proof of this inequality depends on whether or not $x$ and $\tau(x)$ lie in the support of the multiplicity function. In case $\tau(x)$ is not in the support of $m$, the desired inequality follows immediately from the definition of the supplementary function $s(\cdot)$.

Assume next that both $x$ and $\tau(x)$ are in the support of the multiplicity function. Because $\tau$ is cyclic, we shall assume initially that $x=x_{i_{k}}$ where $k<L$. Then $\tau(x)=x_{i_{k+1}}=x_{i_{k}+1}$. But then

$$
\begin{aligned}
s(\tau(x)) & =i_{k+1}-i_{0}-\sum_{j=1}^{k+1} m\left(x_{i_{j}}\right) \\
& =i_{k}+1-i_{0}-\sum_{j=1}^{k} m\left(x_{i_{j}}\right)-m\left(x_{i_{k+1}}\right) .
\end{aligned}
$$

If $k=0$, it follows that

$$
s(\tau(x))=i_{0}-i_{0}+1-m\left(x_{i_{1}}\right) \leqq 0=s\left(x_{i_{0}}\right) \leqq s(x)+1 .
$$

If $k \neq 0$, then we have

$$
s(\tau(x))=s(x)+1-m\left(x_{i_{k+1}}\right) \leqq s(x)+1 .
$$

Now suppose that $x=x_{i_{L}}$ and both $x$ and $\tau(x)$ are in the support of $m$. Then $x_{i_{L}}$ must be $x_{n-1}$ and $\tau(x)=x_{0}$ so both $x_{n-1}$ and $x_{0}$ are in the support of $m$. We know $s(\tau(x))=s\left(x_{0}\right)=0$, so we need to show $s\left(x_{n-1}\right)+1 \geqq 0$. This is done as follows:

$$
s\left(x_{n-1}\right)=(n-1)-0-\sum_{j=1}^{L} m\left(x_{i_{j}}\right)=n-1-\left(n-m\left(x_{0}\right)\right) \geqq 0 .
$$

Thus $s\left(x_{n-1}\right)+1 \geqq 0$ and hence $s\left(x_{0}\right) \leqq s\left(x_{n-1}\right)+1$.

Lastly, assume that $x \notin \operatorname{supp} m$ and $\tau(x) \in \operatorname{supp} m$. In this case $s(x)=s(y)+k$ for some $y$ as in the definition of the function $s(\cdot)$. A computation similar to the preceding one shows that $s(\tau(x)) \leqq$ $s(x)+1$.

Step 3. The proof will be completed by showing that the multiplicity function $m_{1}$ for the two-sided invariant subspace $l^{2}(B)$ is equal to the original function $m$. It follows immediately from 
the definition of the partition that the supports of the functions $m$ and $m_{1}$ are identical. To show $m=m_{1}$, it suffices to identify the $\lambda$-wandering set $A$ and show that $m(x)=\operatorname{card} A_{x}$.

We know that the wandering set is $B \backslash \lambda(B)$. Let

$$
A=\bigcup_{l}\left\{(l, x),(l+1, x), \cdots,(l+m(x)-1, x) \mid x \in E_{l} \cap \operatorname{supp} m\right\} .
$$

We shall show $A=B \backslash \lambda(B)$. Clearly $A \subseteq B$. Suppose $A \cap \lambda(B) \neq \varnothing$. Specifically, we may assume (by invertibility) the point $\left(k+n_{2}, x_{i_{q}}\right)$ in the intersection $A \cap \lambda(B)$ satisfies $\lambda^{\alpha}\left(l+n_{1}, x_{i_{p}}\right)=\left(k+n_{2}, x_{i_{q}}\right)$ where $x_{i_{p}} \in E_{l}, x_{i_{q}} \in E_{k}, 0 \leqq n_{1}<m\left(x_{i_{p}}\right), 0 \leqq n_{2}<m\left(x_{i_{q}}\right)$, and $\alpha$ is a positive integer less than $n$. Observe that

$$
\tau^{\alpha}\left(x_{i_{p}}\right)=x_{i_{q}}\left(\text { so that } \alpha=\left(i_{q}-i_{p}\right)(\bmod n)\right)
$$

and

$$
l+n_{1}+\alpha=k+n_{2} .
$$

Due to the cyclic nature of $\tau$, we are forced into considering separate cases. In each case, the point $\left(k+n_{2}, x_{i_{q}}\right)$ cannotbe in $A \cap \lambda(B)$ and hence this intersection will be empty.

For the first case, suppose $i_{p}<i_{q}$ and $i_{p} \neq i_{0}$. We have

$$
s\left(x_{i_{p}}\right)=i_{p}-i_{0}-\sum_{j=1}^{p} m\left(x_{i_{j}}\right)
$$

and

$$
s\left(x_{i_{q}}\right)=i_{q}-i_{0}-\sum_{j=1}^{q} m\left(x_{i_{j}}\right)
$$

Thus

$$
\begin{aligned}
k-l & =s+k-(s+l) \\
& =s\left(x_{i_{q}}\right)-s\left(x_{i_{p}}\right) \\
& =i_{q}-i_{p}-\sum_{j=p+1}^{q} m\left(x_{i_{\boldsymbol{j}}}\right) \\
& =\alpha-\sum_{j=p+1}^{q} m\left(x_{i_{\boldsymbol{i}}}\right) .
\end{aligned}
$$

Hence $k=\alpha+l-\sum_{j=p+1}^{q} m\left(x_{i_{j}}\right) \neq \alpha+l+n_{1}-n_{2}$ for any $n_{1}, \quad n_{2}$ since $n_{1} \in\left\{0,1,2, \cdots, m\left(x_{i_{p}}\right)-1\right\}$ and $n_{2} \in\left\{0,1,2, \cdots, m\left(x_{i_{q}}\right)-1\right\}$. This contradicts ( $\dagger$ ). So no element in $A \cap \lambda(B)$ can satisfy the hypotheses of this case. The remaining cases are all based on the demonstration of this first case and are left to the reader.

We now have $A \subseteq B \backslash \lambda(B)$. To show $A=B \backslash \lambda(B)$, simply observe that, by construction, the cardinality of the set $A$ is the 
same as that of the set $X$. Since the wandering set $B \backslash \lambda(B)$ has cardinality $n$, we must have $A=B \backslash \lambda(B)$.

Lastly observe that, by construction, $m(x)=\operatorname{card} A_{x}$ for each element $x$ in $X$.

REMARK 3.5. (1) The subspace $l^{2}(B)$ constructed in the proof of the theorem has the property that $l^{2}(B) \subseteq l^{2}\left(\boldsymbol{Z}_{+} \times X\right)$ but $L_{\delta}^{-1} l^{2}(B) \nsubseteq$ $l^{2}\left(Z_{+} \times X\right)$. Such a subspace is said to be left-justified. This is equivalent of course to the property that $B \cap(\{0\} \times X) \neq \varnothing$ but $B \cap(\{-1\} \times X)=\varnothing$.

(2) Clearly a multiplicity function for a two-sided invariant subspace can be identified with an ordered $n$-tuple of nonnegative integers $\left(\alpha_{0}, \alpha_{1}, \cdots, \alpha_{n-1}\right)$ with the property that $\alpha_{0}+\alpha_{1}+\cdots+$ $\alpha_{n-1}=n$. The number of distinct $n$-tuples with this property (and hence the number of distinct left-justified two-sided invariant subspaces $\left.l^{2}(B)\right)$ is $\left(\begin{array}{c}2 n-1 \\ n\end{array}\right)=(2 n-1) ! /(n-1) ! n !,[8$, p. 139]. In $\S 4$ we shall show how to use this finite collection of subspaces as canonical models for all left-pure invariant subspaces for the algebra $\mathfrak{R}_{+}$.

The final theorem of this section illustrates how to obtain a multiplicity function directly from a two-sided invariant subspace of the form $l^{2}(B)$. Recall that the multiplicity function of such a subspace was defined using a decomposition of the projection onto the wandering subspace.

THEOREM 3.6. Let $l^{2}(B)$ be a (nontrivial) two-sided invariant subspace with associated ordered partition $\left\{E_{k}\right\}_{k=-\infty}^{\infty}$ (cf. 3.1.3). Then the multiplicity function for the subspace $l^{2}(B)$ can be formed as follows: for $x$ in $E_{l}, m(x)=\min _{k}\left\{n_{k}+k-l \mid n_{k}\right.$ is the first positive integer with the property that $\left.x \in \tau^{n_{k}}\left(E_{k}\right) ; k \in Z\right\}$.

Proof. Recall that $B=\bigcup_{k \in Z} \boldsymbol{Z}_{(k)} \times E_{k}$ and there is an integer $N$ such that $E_{k}=\varnothing, k<N$ (cf. Remark 3.1). Note next that $m$ as defined above is a nonnegative-valued function since the partition $\left\{E_{k}\right\}$ has the property that $\tau\left(E_{k}\right) \subseteq \bigcup_{l \leqq k+1} E_{l}$. We will assume throughout the proof that the element $x$ is in $E_{l}$. Let $A=B \backslash \lambda(B)$ be the wandering set for the subspace and recall that we need only show that $m(x)$ is the cardinality of the set $A_{x}$, the $x$-section of the set $A$.

We first show that the equation $m(x)=0$ is equivalent to the equation $A_{x}=\varnothing$. Suppose that $m(x)=0$. Then there exists an integer $k$ such that $n_{k}+k=l$ (so that in particular $k<l$ ) and 
hence $x$ is in $\tau^{n_{k}}\left(E_{l_{k}}\right)$. But then $\tau^{-n_{k}} x$ is an element of the set $E_{k}$ so that the point $\left(k, \tau^{-n_{k}} x\right)$ is in $B$. We now have $\lambda^{n_{k}}\left(k, \tau^{-n_{k}} x\right)=$ $(l, x)$ so that the point $(l, x)$ is not in $A_{x}$. Hence $A_{x}$ must be empty since if $A_{x}$ is to be nonempty, it must contain at least the point $(l, x)$ (along with possibly other elements of the form $(l+j, x)$ for $j>0)$.

On the other hand if $A_{x}$ is empty then $(l, x)$ is in $B$ but not in $A$. Thus $(l, x)$ must be in $\lambda(B)$ and hence $\lambda^{-1}(l, x)=\left(l-1, \tau^{-1} x\right)$ is in $B$. By left-invariance and the definition of the partition, $(l-2$, $\left.\tau^{-1} x\right) \notin B$. Thus $n_{l-1}=1$ so that the smallest positive integer $\alpha$ such that $x$ is in $\tau^{\alpha}\left(E_{l-1}\right)$ is $\alpha=1$. Thus $0 \in\left\{n_{k}+k-l \mid n_{k}\right.$ is the first positive integer with the property that $\left.x \in \tau^{n_{k}}\left(E_{k_{k}}\right)\right\}$ and hence $m(x)=0$.

Assume now that $m(x)$ is different from zero. Let $j$ be an integer such that $0 \leqq j<m(x)$ and set $n_{0}=l+j$. Suppose the point $\left(n_{0}, x\right)$ does not belong to $A_{x}$. This means that $\left(n_{0}, x\right)=\lambda\left(n_{0}-\right.$ $\left.1, \tau^{-1} x\right)$ with $\left(n_{0}-1, \tau^{-1} x\right)$ in $B$. Then $\tau^{-1} x$ lies in $E_{k}$ for some $k \leqq$ $n_{0}-1$. Hence $m(x) \leqq n_{l}+k-l \leqq 1+\left(n_{0}-1\right)-l=j<m(x)$. This contradiction shows that $\left(n_{0}, x\right)$ lies in $A$ and hence $m(x) \leqq \operatorname{card} A_{x}$.

Finally the point $(l+m(x), x)$ is in $B$. Let $n_{k}$ and $k$ be the integers as in the statement of the theorem so that $x$ is in $\tau^{n_{k}}\left(E_{k}\right)$ and $m(x)=n_{k}+k-l$. Then $(l+m(x), x)=\left(n_{k}+k, x\right)=\lambda^{n_{k}}(k$, $\left.\tau^{-n_{k}} x\right)$ and the point $\left(k, \tau^{-n_{k}} x\right)$ is in $B$ since $\tau^{-n_{k}} x$ is in $E_{k}$. Thus $(l+m(x), x)$ is in $B$ but not in $A$ so that $(l+m(x), x)$ is not in $A_{x}$. This completes the proof that $m(x)=\operatorname{card} A_{x}$.

4. Canonical models. In this section we show how to use the finite number of left-justified $\mathfrak{L}_{+} \vee \Re_{+}$-invariant subspaces of the form $l^{2}(B)$ as canonical models for the pure $\mathbb{L}_{+}$-invariant subspaces.

TheORem 4.1. Let $\mathscr{C l}$ be a nontrivial left-pure invariant subspace of $l^{2}(\boldsymbol{Z} \times X)$. Then there exists a two-sided invariant subspace of the form $l^{2}(B)$ and a partial isometry $R_{v}$ in the algebra $\Re$ such that $P_{\text {" }}=R_{v} P_{l^{2}(B)} R_{v}^{*}$ and hence $\mathscr{C}=R_{v} l^{2}(B)$.

Proof. Let $\mathscr{F}$ be the left-wandering subspace associated with $\mathscr{C}\left(\mathscr{F}=\mathscr{C} \ominus L_{\hat{o}} \mathscr{C}\right)$. The dimension of $\mathscr{F}$ is less than or equal to $n$. Let $m(\cdot)$ be the multiplicity function for $\mathscr{C l}$. If $\sum_{x \in X} m(x)=$ $n$, then there exists a left-justified two-sided invariant subspace $l^{2}(B)$ with multiplicity function $m$. The theorem now follows from Theorem 3.4 in [5] and, in fact, $R_{v}$ is a unitary operator in this case.

If $\sum_{x \in X} m(x)<n$, then there exists an element $x_{k}$ in $X$ such that $m\left(x_{k}\right)=0$. Define a new function $m_{1}$ by 


$$
m_{1}(x)=\left\{\begin{array}{cl}
m(x) & x \neq x_{k}, \\
n-\sum_{x \in X} m(x) & x=x_{k}
\end{array}\right.
$$

Then $m_{1}$ is a multiplicity function for a left-justified two-sided invariant subspace $l^{2}(B)$. Let $\mathscr{F}_{1}=l^{2}(B) \ominus L_{\delta} l^{2}(B)$, the wandering subspace associated with $l^{2}(B)$, and let $P_{\mathscr{F}_{1}}=\sum_{x} \oplus P_{1}(x)$ (cf. Remark 3.1). By construction rank $P_{1}(x)=m_{1}(x)$. Now let $Q=\sum_{x \neq x_{k}} \oplus P_{1}(x)$. We may also decompose the projection onto the wandering subspace for $\mathscr{C}: P_{\mathscr{F}}=\sum_{x \in X} \oplus P(x)$. It follows that the projection $Q$ is equivalent to the projection $P_{\mathscr{F}}$ in $\mathfrak{M}_{L}^{\prime}$ since the corresponding dimensions are identical (see [2] for a discussion of equivalence of projections). Since $Q<P_{\mathscr{F}_{1}}$, it follows that $P_{\mathscr{F}} \prec P_{\mathscr{F}_{1}}$ in $\mathfrak{M}_{L}^{\prime}$. So by Theorem 3.4 of [5], there exists a partial isometry $R_{v}$ in $\Re$ such that $P_{. /}=R_{v} P_{l^{2}(B)} R_{v}^{*}$ and this completes the proof.

As a corollary, we are able to show that any two-sided invariant subspace that is not left-reducing is (left) pure and full. Lemma 4.2 will be useful for the proof. Let $L^{p}$ and $H^{p}$ denote the usual Lebesgue and Hardy spaces on the circle with normalized Lebesgue measure. Let $H_{0}^{p}$ denote the space of $H^{p}$-functions vanishing at the origin. Recall that Szego's theorem asserts that if $\omega$ is a positive integrable function on the circle, $T$, then the inf $\int_{T}|1-f|^{2} \omega d m$, which is taken over all polynomials $f$ vanishing at zero, is precisely $\exp \left(\int \log \omega d m\right)$ where the expression is zero by fiat if $\log \omega$ is not integrable. It follows from this fact that if $f$ is an $L^{2}$-function then the distance in $L^{2}$ from $f$ to the closed subspace $\overline{\left(f H_{0}^{\infty}\right)}$ is

$$
\exp \left(\int \log |f|^{2} d m\right)
$$

LEMMA 4.2. Let $E$ be a measurable subset of the circle such that both $E$ and its complement $\boldsymbol{T} \backslash E$ have positive measure. Then the closure of $1_{E} H^{2}$ in $L^{2}, \overline{1_{E} H^{2}}$, equals $1_{E} L^{2}$.

Proof. From Szego's theorem we know that the distance from the indicator function $1_{E}$ to the space $1_{E} H_{0}^{2}$ is $\exp \left(\int \log 1_{E} d m\right)$, which equals zero since $1_{E}$ vanishes on a set of positive measure. Thus $1_{E} \in \overline{1_{E} H_{0}^{2}}$. From this it is easy to see that the subspace $\overline{1_{E} H^{2}}$ is a reducing subspace for the operator of multiplication by $z$ (i.e., the shift operator). Thus by [7] there exists a measurable subset $F$ such that $\overline{1_{E} H^{2}}=1_{F} L^{2}$. It is easy to show that the sets $E$ and $F$ differ by at most a null set so that $\overline{1_{E} H^{2}}=1_{E} L^{2}$. 
Corollary 4.3. Let $\mathscr{C}$ be a two-sided invariant subspace that is not left-reducing. Then $\mathscr{K}$ is (left) pure and full.

Proof. We shall assume first that $\mathscr{C}$ is (left) pure and show that $\mathscr{M}$ is full. Subsequent to this we shall show how this assumption can be replaced by the assumption that $\mathscr{C}$ is not left-reducing.

Since $\mathscr{C}$ is a pure subspace we can apply Theorem 4.1 and write $\mathscr{C}=R_{v} l^{2}(B)$ where $R_{v}$ is a partial isometry in the algebra $\Re$ and $l^{2}(B)$ is a two-sided invariant subspace.

Let $P$ be the final projection of $R_{v}$ so that $P=R_{v} R_{v}^{*}$ is an element of $\Re$. In this proof we shall use the notation $L^{2}$ to refer to $l^{2}(Z \times X)$. Thus $P L^{2}=R_{v} L^{2}=R_{v} \mathrm{~V}_{n \leqq 0} L_{\delta}^{n} l^{2}(B)=\mathrm{V}_{n \leqq 0} L_{\delta}^{n} R_{v} l^{2}(B)=$ $\mathrm{V}_{n \leq 0} L_{\delta}^{n} \mathscr{M}$. Observe also that $P$ commutes with $\Re$. To see this let $R_{\varphi}$ be an element of $\mathfrak{M}_{R}$. Then $R_{\varphi} P L^{2}=R_{\varphi} \mathrm{V}_{n \leqq 0} L_{\dot{\delta}}^{n} \mathscr{M}=\mathrm{V}_{n \leqq 0} L_{i}^{n} R_{\varphi} \mathscr{C} \cong$ $\mathrm{V}_{n \leq 0} L_{\delta}^{n} \mathscr{M}=P L^{2}$. So $P L^{2}$ is invariant for $\mathfrak{M}_{R}$ and hence the projection $P$ is in the commutant of $\mathfrak{M}_{R}$. To show $P$ commutes with $R_{\delta}$ observe that $R_{\delta} P R_{\delta}^{*} L^{2}=R_{\delta} P L^{2} \leqq P L^{2}$ so that $R_{\delta} P R_{\delta}^{*} \leqq P$. But since the von Neumann algebra $\Re$ is finite and the projections $R_{\hat{\delta}} P R_{\delta}^{*}$ and $P$ are equivalent in $\Re$, we must have $R_{\hat{\delta}} P R_{\hat{\delta}}^{*}=P$. Thus $P$ is in $\Re^{\prime}$, the commutant of $\Re$. But $\Re^{\prime}=\mathbb{R}$ and so $P$ lies in $\mathscr{Z}(\Re)$, the center of $\Re$ (which is also $\mathscr{Z}(\mathfrak{R})$, the center of $\mathfrak{L}$ ).

By Corollary 2.2 we can represent $P$ as an operator of the form $1_{E} \otimes I$ acting on the Hilbert space $L^{2}(T) \otimes M_{n}$. If $E$ is almost all of the circle, we may take $P$ to be the identity and it follows that $\mathscr{C}$ is full. If $\mu(T \backslash E)>0$ so that the indicator function $1_{E}$ vanishes on a set of positive measure, we show that $\mathscr{M}$ is not pure, contrary to our assumption.

First note that $P$ is also the initial projection of $R_{v}$ since $\Re$ is a finite von Neumann algebra and $P$ is a central projection. It is easy to see that we can choose an integer $N>0$ such that $L_{i}^{N} W^{-1}\left(H^{2} \otimes M_{n}\right) \subseteq l^{2}(B)$ where $W$ is the isomorphism of $\S 2$. Thus we have

$$
\begin{aligned}
P L^{2} & \supseteq \mathscr{M} \\
& =R_{v} P l^{2}(B) \quad(P \text { is the initial projection }) \\
& \supseteq R_{v} P L_{\delta}^{N} W^{-1}\left(H^{2} \otimes \boldsymbol{M}_{n}\right) \\
& =R_{v} L_{\delta}^{N} P W^{-1}\left(H^{2} \otimes \boldsymbol{M}_{n}\right) \\
& =R_{v} L_{\delta}^{N} W^{-1}\left(W P W^{-1}\right)\left(H^{2} \otimes \boldsymbol{M}_{n}\right) \\
& =R_{v} L_{\delta}^{N} W^{-1}\left(\overline{\left.1_{E} \otimes I\right)\left(H^{2} \otimes M_{n}\right.}\right) \\
& =R_{v} L_{\delta}^{N} W^{-1}\left(\overline{1_{E} H^{2}} \otimes M_{n}\right) \\
& =R_{v} L_{\delta}^{N} W^{-1}\left(1_{E} L^{2}(\boldsymbol{T}) \otimes \boldsymbol{M}_{n}\right) \quad(4.2) \\
& =R_{v} L_{\delta}^{N} W^{-1}\left(1_{E} \otimes I\right)\left(L^{2}(\boldsymbol{T}) \otimes \boldsymbol{M}_{n}\right)
\end{aligned}
$$




$$
\begin{aligned}
& =R_{v} L_{\delta}^{N} P L^{2} \\
& =P R_{v} L_{\delta}^{N} L^{2} \\
& =P L^{2} \quad(P \text { is the final projection). }
\end{aligned}
$$

But this contradicts the purity of $\mathfrak{M}$ since $P L^{2}$ is a reducing subspace. This completes the proof under the assumption that $\mathscr{M}$ is a pure subspace.

Now assume that $\mathscr{C}$ is a two-sided invariant subspace that is not left-reducing. To complete the proof it suffices to show that $\mathscr{C l}$ is pure. We shall show that if $\mathscr{C}$ is not pure then, in fact, $\mathscr{C}$ must reduce $\mathfrak{R}_{+}$. We may break $\mathscr{C}$ into its reducing and pure pieces: $\mathscr{C}=\mathscr{M}_{1} \oplus \mathscr{L}_{2}$ where $\mathscr{M}_{1}=\bigcap_{n \geq 0} L_{\delta}^{n} \mathscr{C l}$ and $\mathscr{M}_{2}=$ $\sum_{n \geq 0} L_{\dot{\delta}}^{n} \mathscr{F}$ ([5], Prop. 3.3; or [7]). If $\mathscr{M}$ is not a pure subspace then $\mathscr{M}_{1} \neq\{0\}$. The subspace $\mathscr{H}_{1}$ is left-reducing; in addition, $\mathscr{M}_{1}$ is right-reducing. To see this we need only show $\mathscr{A}_{1}$ reduces $R_{\delta}$. Since $\mathscr{A}_{1}$ reduces $\mathcal{L}$, we have $\mathscr{M}_{1}=R_{p} L^{2}$ where $R_{p}$ is a projection in $\Re$, the commutant of $\mathbb{R}$. (Recall $L^{2}=l^{2}(Z \times X)$.) But since $\mathscr{K}_{1}$ is invariant for $R_{\hat{o}}, R_{\hat{\delta}} R_{p} R_{\hat{\delta}}^{*} L^{2}=R_{\delta} R_{p} L^{2} \leqq R_{p} L^{2}$. Thus $R_{\delta} R_{p} R_{\delta}^{*} \leqq R_{p}$ and so $R_{\delta} R_{p} R_{\delta}^{*}=R_{p}$ as in the first part of the proof. It follows that $R_{p}$ commutes with $\Re$ and so $R_{p} \in \mathbb{R} \cap \mathfrak{R}=\mathfrak{L} \cap \mathfrak{L}^{\prime}=\mathscr{Z}(\mathbb{R})$, the center of 2 . The projection $R_{p}$ may be represented as $1_{E} \otimes I$ acting on $L^{2}(\boldsymbol{T}) \otimes M_{n}$. Since $\mathscr{A}_{2}$, the pure part of $\mathscr{M}$, is orthogonal to $\mathscr{A}_{1}, W \mathscr{A}_{2}$ must lie in the range of $1_{E^{\prime}} \otimes I\left(E^{\prime}=T \backslash E\right)$. Hence $W \mathscr{A}_{2} \subseteq L^{2}\left(E^{\prime}\right) \otimes M_{n}$. Representing $W \mathscr{M}_{2}$ as a matrix of subspaces, $W \mathscr{A}_{2}=\left[\mathscr{M}_{i j}\right]$, we have $\mathscr{N}_{i j} \subseteq L^{2}\left(E^{\prime}\right)$ for each $i, j$.

Since $\mathscr{C}_{2}$ is invariant under $L_{\hat{j}}$, it is easy to see that each subspace $\mathscr{N}_{i j}$ is invariant under multiplication by $\chi_{1}$, the bilateral shift on $L^{2}(\boldsymbol{T})$. Using the fact that $\mathscr{M}_{2}$ is pure, a calculation shows that the subspaces $\mathscr{M}_{i j}$ do not reduce the shift, unless $\mathscr{M}_{i j}=\{0\}$. Hence for each $i, j$, either $\mathscr{M}_{i j}=\varphi_{i j} H^{2}(\boldsymbol{T})\left(\left|\varphi_{i j}\right|=1\right.$ a.e. $)$ or $\mathscr{M}_{i j}=\{0\}$. But since $\mathscr{N}_{i j} \subseteq L^{2}\left(E^{\prime}\right)$, we conclude that $\mathscr{M}_{i j}=\{0\}$ for all $i, j$. Hence $\mathscr{A}_{2}=\{0\}$ and so $\mathscr{C}$ is a (left) reducing subspace.

REMARK 4.4. The preceding proof shows that a two-sided invariant subspace is either reducing or pure. Thus in the decomposition $\mathscr{M}=\mathscr{M}_{1} \oplus \mathscr{M}_{2}$ as above, at most one of the spaces $\mathscr{M}_{1}$, $\mathscr{M}_{2}$ is a nonzero subspace.

5. Uniqueness. In this last section, we comment on the degree of nonuniqueness in the construction of canonical models. Theorem 4.1 not only states that if $\mathscr{C}$ is a left-pure invariant subspace then $\mathscr{C}=R_{v} l^{2}(B)$, but its proof and the proof of Theorem 3.4 actually construct the two-sided invariant subspace $l^{2}(B)$. Since $\mathscr{M}$ also equals $R_{v} R_{\tilde{o}}^{-k} l^{2}\left(\rho^{k}(B)\right)=R_{w} l^{2}(C)$, the subspace $l^{2}(B)$ is not unique. 
However we can choose a specific subspace as follows.

For any multiplicity function such that $\sum m(x)=n$, construct the left-justified two-sided invariant subspace $l^{2}(B)$ as in the proof of 3.4. In case $\sum m(x)<n$, extend the function to another multiplicity function $m_{1}$ by defining $m_{1}\left(x_{k}\right)=n-\sum_{x \neq x_{k}} m(x)$, where $x_{k}$ is the element of $X$ outside the support of $m$ with the smallest index $k$. Now construct the subspace $l^{2}(B)$ for this multiplicity function as in 3.4. Using this procedure, any given multiplicity function will yield a unique left-justified two-sided invariant subspace of the form $l^{2}(B)$. It remains only to consider the partial isometry in the expression $\mathscr{C}=R_{v} l^{2}(B)$. The following proposition is a reformulation of a corollary found in [3, p. 64].

Proposition 5.1. Let $U$ be a bilateral shift on a Hilbert space $\mathscr{H}$, let $\mathscr{H}_{+}$be a full, pure invariant subspace for $U$, and let $\mathscr{L}$ be a pure invariant subspace for $U$. If $V_{1}$ and $V_{2}$ are partial isometries on $\mathscr{H}$ which commute with $U$ and satisfy $V_{i} P_{\mathscr{C}_{+}} V_{i}^{*}=$ $P_{\mathscr{M}}$, then there is a partial isometry $W$ on $\mathscr{H}$ such that: (1) the initial space of $W$ is the initial space of $V_{2}$; (2) the final space of $W$ is the initial space of $V_{1}$; (3) $W$ commutes with $U$; (4) $W$ is reduced by $\mathscr{K}_{+}$; and (5) $V_{2}=V_{1} W$.

Proof. Let $\mathscr{F}=\mathscr{K}_{+} \ominus U \mathscr{K}_{+}$and $\mathscr{G}=\mathscr{C} \ominus U \mathscr{M}$. Then $V_{i} P_{\mathscr{T}} V_{i}^{*}=V_{i}\left(P_{\mathscr{C}_{+}}-U P_{\mathscr{C}_{+}} U^{*}\right) V_{i}^{*}=V_{i} P_{{ }^{\prime}+} V_{i}^{*}-U V_{i} P_{\mathscr{C}} V_{i}^{*} U^{*}=P_{\mathscr{M}}-$ $U P_{\mathscr{A}} U^{*}=P_{\mathscr{S}}$. Thus when restricted to $\mathscr{F}$, each $V_{i}$ is a partial isometry mapping onto $\mathscr{G}$. For $i=1,2$, let $\mathscr{F}_{i}$ be the initial space of $V_{i} \mid \mathscr{F}_{i}$. Then $\mathscr{F}_{i}$ is a subspace of $\mathscr{F}$ and $V_{i}$ maps $\mathscr{F}_{i}$ isometrically onto $\mathscr{G}$. Because $V_{i}$ commutes with $U$ and $\mathscr{F}_{i}$ is contained in the complete wandering subspace $\mathscr{F}$, it is easy to check that the initial space of $V_{i}$ is $\sum_{n=-\infty}^{\infty} \oplus U^{n} \mathscr{F}_{i}$. Since $V_{i} \mathscr{F}_{i}=\mathscr{G}$, we can find a partial isometry $W_{0}$ mapping $\mathscr{F}$ to $\mathscr{F}$ such that the initial space of $W_{0}$ is $\mathscr{F}_{2}$, the final space of $W_{0}$ is $\mathscr{F}_{1}$, and $\left(V_{1} \mid \mathscr{F}_{1}\right) W_{0}=\left(V_{2} \mid \mathscr{F}_{2}\right)$. Define $W$ on all of $\mathscr{H}=\sum_{n=-\infty}^{\infty} U^{n} \mathscr{F}$ by the following formula

$$
W\left(\sum U^{n} e_{n}\right)=\sum U^{n} W e_{n}
$$

where $\left\{e_{n}\right\}_{n=-\infty}^{\infty}$ is a sequence in $\mathscr{F}$ satisfying $\sum\left\|e_{n}\right\|^{2}<\infty$. (For details on this definition see the proof of Theorem 3.4 in [5]. It is helpful to note that if the spaces $U^{n} \mathscr{F}$ are identified and operators on $\mathscr{H}$ are written as operator matrices, $W$ is $\operatorname{diag}\left(\cdots, W_{0}, W_{0}\right.$, $\left.W_{0}, \cdots\right)$.) It is immediate that $W$ satisfies the conclusions of the proposition.

Our uniqueness theorem is a simple translation of Proposition 
5.1. We include a precise statement for completeness.

COROLLARY 5.2. Let $\mathscr{C}$ be a left-pure invariant subspace and let $l^{2}(B)$ be a two-sided invariant subspace. If $R_{v_{1}}$ and $R_{v_{2}}$ are partial isometries in $\Re$ which satisfy $R_{v_{i}} P_{l^{2}(B)} R_{v_{i}}^{*}=P_{\mathscr{A}}$, then there is a partial isometry $R_{w}$ in $\Re$ whose initial space is the initial space of $R_{v_{2}}$, whose final space is the initial space of $R_{v_{1}}$, which is reduced by $l^{2}(B)$, and which satisfies $R_{v_{2}}=R_{v_{1}} R_{w}$.

Proof. Let $\widetilde{W}$ be the partial isometry obtained from Proposition 5.1 for the bilateral shift $L_{\delta}$. We need only show that $\widetilde{W}$ is in $\Re$. But since the initial space of $R_{v_{1}}$ is the final space of $\widetilde{W}$, we have $R_{v_{1}}^{*} R_{v_{1}}=R_{v_{1}}^{*} R_{v_{1}} \widetilde{W}=\widetilde{W}$ and hence $\widetilde{W}$ is in $\Re$.

The preceding corollary does not answer completely all questions of uniqueness. In particular, it does not describe the partial isometries in $\Re$ that are reduced by a canonical model. A satisfactory description can be given in case the subspace of Corollary 5.2 is both full and pure. In this case $\mathscr{C}=R_{u} l^{2}(B)$ where $R_{u}$ is a unitary operator in $\Re$. The task is to describe those unitary operators in $\Re$ that are reduced by a particular canonical model. Expressing $R_{u}$ in its matricial form as discussed in $\S 2$, we can show that $R_{u}$ has a specific form, which depends on the canonical model reducing it. The following two examples illustrate this.

EXAMPLE 5.3. Consider the subspace $l^{2}\left(\boldsymbol{Z}_{+} \times X\right)$ and let $P$ be the projection onto the subspace. It is easy to show that $\Re \cap\{P\}^{\prime}=$ $\mathfrak{R}_{+} \cap \mathfrak{R}_{+}^{*}=\mathfrak{M}_{R}$. The image of $\mathfrak{M}_{R}$ in $L^{\infty}(\boldsymbol{T}) \otimes \boldsymbol{M}_{n}$ is the algebra of (right multiplications by) diagonal matrices with constant entries along the diagonal. Thus the unitary operators in $\Re$ that reduce $l^{2}\left(\boldsymbol{Z}_{+} \times X\right)$ can be represented as right multiplications on $L^{2}(\boldsymbol{T}) \otimes \boldsymbol{M}_{n}$ by matrices of the form

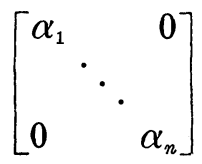

with $\left|\alpha_{i}\right|=1$.

EXAMPLE 5.4. A more typical example can be obtained by considering the case that $X$ has cardinality 3 . Let $B=\left\{\left(0, x_{2}\right),\left(1, x_{0}\right)\right.$, $\left.\left(1, x_{2}\right)\right\} \cup\left(\boldsymbol{Z}_{(2)} \times X\right)$ and consider the subspace $l^{2}(B)$. The image of this (two-sided invariant) subspace in $L^{2}(\boldsymbol{T}) \otimes M_{n}$ (under the isomorphism discussed in $\S 2$ ) is 


$$
\left[\begin{array}{lll}
H_{0}^{2} & H_{0}^{2} & H_{1}^{2} \\
H^{2} & H^{2} & H_{0}^{2} \\
H^{2} & H^{2} & H_{0}^{2}
\end{array}\right] .
$$

(Recall $H_{0}^{2}=\chi_{1} H^{2}$ and $H_{1}^{2}=\chi_{2} H^{2}$.) A computation shows that if $R_{u}$ is a unitary operator in $\Re$ reduced by $l^{2}(B)$, then $R_{u}$ must be right multiplication by a matrix of the form

$$
\left[\begin{array}{lll}
a_{11} & a_{12} & a_{13} \chi_{1} \\
a_{21} & a_{22} & a_{23} \chi_{1} \\
a_{31} \bar{\chi}_{1} & a_{32} \bar{\chi}_{1} & a_{33}
\end{array}\right]
$$

with the constants $a_{i j}$ chosen so that the matrix is unitary (recall $\left.\chi_{k}(z)=z^{k}\right)$.

This second example exhibits the general characteristics of the form of a unitary operator reduced by a canonical model. In general if we represent $R_{u}$ as right multiplication by the matrix $\left[\varphi_{i j}\right.$ ] on $L^{2}(\boldsymbol{T}) \otimes \boldsymbol{M}_{n}$, one can show that each $\varphi_{i j}$ has the form $c_{i j} \chi_{k}$ where $c_{i j}$ is a complex constant and $k$ is $-1,0$, or 1 . In particular $\varphi_{i i}$ can be shown always to be a constant; if $i>j, \varphi_{i j}=c_{i j} \chi_{k}$ and $k=0$ or 1 ; and if $i<j$ then $\varphi_{i j}=c_{i j} \chi_{k}$ and $k=0$ or -1 . Moreover one can construct a simple algorithm, based on the form of the canonical model for deciding the value of $k$. Although we feel that these results are of value, their istatements and proofs are notationally cumbersome and so are omitted.

\section{REFERENCES}

1. M. B. Abrahamse and R. G. Douglas, Subnormal opaerators related to multiply connected domains, Advances in Math., 19 (1976), 106-148.

2. J. Dixmier, Les algèbres d'opérateurs dans l'espace Hilbertien, Gauthier-Villars, Paris, 1969.

3. H. Helson, Lectures on invariant subspaces, Academic Press, London and New York, 1964.

4. - Analyticity on Compact Abelian Groups, in Algebras in Analysis, Academic Press, New York, 1975.

5. M. McAsey, Invariant subspaces of non-self-adjoint crossed products, Pacific J. Math., 248 (1979), 381-409.

6. M. McAsey, P. S. Muhly and K.-S. Saito, Non-self-adjoint crossed products, Trans. Amer. Math. Soc., (to appear).

7. H. Radjavi and P. Rosenthal, Invariant subspaces, Springer-Verlag, New York, Heidelberg and Berlin, 1973.

8. E. M. Stein and G. Weiss, Introduction to Fourier analysis on Euclidean spaces, Princeton University Press, Princeton, 1971.

Received April 20, 1979. Portions of this paper are taken from the author's doctoral dissertation written at the University of Iowa under the supervision of Paul S. Muhly. 



\section{PACIFIC JOURNAL OF MATHEMATICS}

\section{EDITORS}

DONALD BABBITT (Managing Editor)

University of California

Los Angeles, CA 90024

Hugo RossI

University of Utah

Salt Lake City, UT 84112

C. C. MOORE and ANDREW OGG

University of California

Berkeley, CA 94720

\section{J. DugundjI}

Department of Mathematics

University of Southern California

Los Angeles, CA 90007

R. FinN and J. Milgram

Stanford University

Stanford, CA 94305

\section{ASSOCIATE EDITORS}
E. F. BeCKENBACH
B. H. Neumann
F. WoLF
K. YoSHIDA

\section{SUPPORTING INSTITUTIONS}

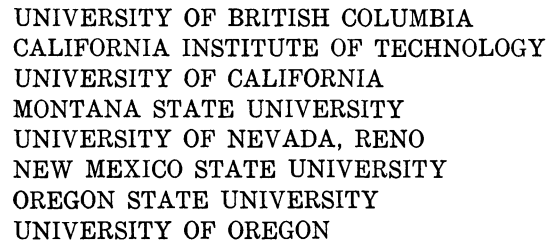

UNIVERSITY OF BRITISH COLUMBIA CALIFORNIA INSTITUTE OF TECHNOLOGY UNIVERSITY OF CALIFORNIA MONTANA STATE UNIVERSITY UNIVERSITY OF NEVADA, RENO NEW MEXICO STATE UNIVERSITY OREGON STATE UNIVERSITY UNIVERSITY OF OREGON

\author{
UNIVERSITY OF SOUTHERN CALIFORNIA \\ STANFORD UNIVERSITY \\ UNIVERSITY OF HAWAII \\ UNIVERSITY OF TOKYO \\ UNIVERSITY OF UTAH \\ WASHINGTON STATE UNIVERSITY \\ UNIVERSITY OF WASHINGTON
}

The Supporting Institutions listed above contribute to the cost of publication of this Journal, but they are not owners or publishers and have no responsibility for its content or policies.

Mathematical papers intended for publication in the Pacific Journal of Mathematics should be in typed form or offset-reproduced, (not dittoed), double spaced with large margins. Please do not use built up fractions in the text of the manuscript. However, you may use them in the displayed equations. Underline Greek letters in red, German in green, and script in blue. The first paragraph or two must be capable of being used separately as a synopsis of the entire paper. Please propose a heading for the odd numbered pages of less than 35 characters. Manuscripts, in triplicate, may be sent to any one of the editors. Please classify according to the scheme of Math. Reviews, Index to Vol. 39. Supply name and address of author to whom proofs should be sent. All other communications should be addressed to the managing editor, or Elaine Barth, University of California, Los Angeles, California, 90024.

50 reprints to each author are provided free for each article, only if page charges have been substantially paid. Additional copies may be obtained at cost in multiples of 50 .

The Pacific Journal of Mathematics is issued monthly as of January 1966. Regular subscription rate: $\$ 84.00$ a year (6 Vols., 12 issues). Special rate: $\$ 42.00$ a year to individual members of supporting institutions.

Subscriptions, orders for numbers issued in the last three calendar years, and changes of address shoud be sent to Pacific Journal of Mathematics, P.O. Box 969, Carmel Valley, CA 93924, U.S.A Old back numbers obtainable from Kraus Periodicals Co., Route 100, Millwood, NY 10546.

PUBLISHED BY PACIFIC JOURNAL OF MATHEMATICS, A NON-PROFIT CORPORATION

Printed at Kokusai Bunken Insatsusha (International Academic Printing Co., Ltd.). 8-8, 3-chome, Takadanobaba, Shinjuku-ku, Tokyo 160, Japan. 


\section{Pacific Journal of Mathematics}

\section{Vol. 91, No. 2 December, 1980}

Victor P. Camillo and Julius Martin Zelmanowitz, Dimension modules ... . . 249

Yonina S. Cooper, Stable sequences in pre-abelian categories ........... 263

Chandrakant Mahadeorao Deo and H. Ship-Fah Wong, On Berry-Esseen approximation and a functional LIL for a class of dependent random fields.........................................

H. P. Dikshit and S. N. Dubey, $|C, 1|$ summability of series associated with

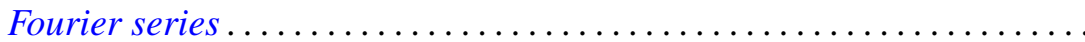

M. Edelstein, On the homomorphic and isomorphic embeddings of a semiflow into a radial flow.

Gilles Godefroy, Compacts de Rosenthal ..................... 293

James Guyker, Commuting hyponormal operators ................ 307

Thomas Eric Hall and Peter R. Jones, On the lattice of varieties of bands of

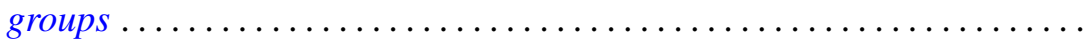

Taqdir Husain and Saleem H. Watson, Topological algebras with orthogonal Schauder bases ....................................

V. K. Jain, Some expansions involving basic hypergeometric functions of two variables. . .

Joe W. Jenkins, On group actions with nonzero fixed points ........... 363

Michael Ellsworth Mays, Groups of square-free order are scarce ........ 373

Michael John McAsey, Canonical models for invariant subspaces... 377

Peter A. McCoy, Singularities of solutions to linear second order elliptic partial differential equations with analytic coefficients by approximation methods...

Terrence Millar, Homogeneous models and decidability.

Stephen Carl Milne, A multiple series transformation of the very well poised

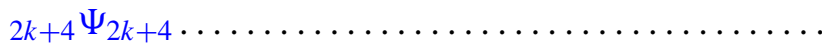

Robert Olin and James E. Thomson, Irreducible operators whose spectra are spectral sets...

Robert John Piacenza, Cohomology of diagrams and equivariant singular

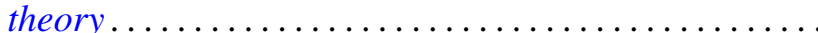

Louis Jackson Ratliff, Jr., Integrally closed ideals and asymptotic prime divisors

Robert Breckenridge Warfield, Jr., Cancellation of modules and groups and stable range of endomorphism rings.................

B. J. Day, Correction to: "Locale geometry" ...............

Stanley Stephen Page, Correction to: "Regular FPF rings" ... 